\title{
Images of the month: Unexpected neurological comorbidity: Occam's razor or Hickam's dictum?
}

\author{
Authors: Josef G Heckmann, ${ }^{A}$ Michael White ${ }^{B}$ and Stefan Ernst ${ }^{C}$
}

Fig 1. Axial cranial computed tomography in an 87-year-old woman. a) Ischaemic infarction in the territory of the left posterior cerebral artery (white arrows). b) Calcified meningioma with prominent perifocal oedema in the right occipital (black arrows) and a left-sided chronic subdural haematoma (white arrows).
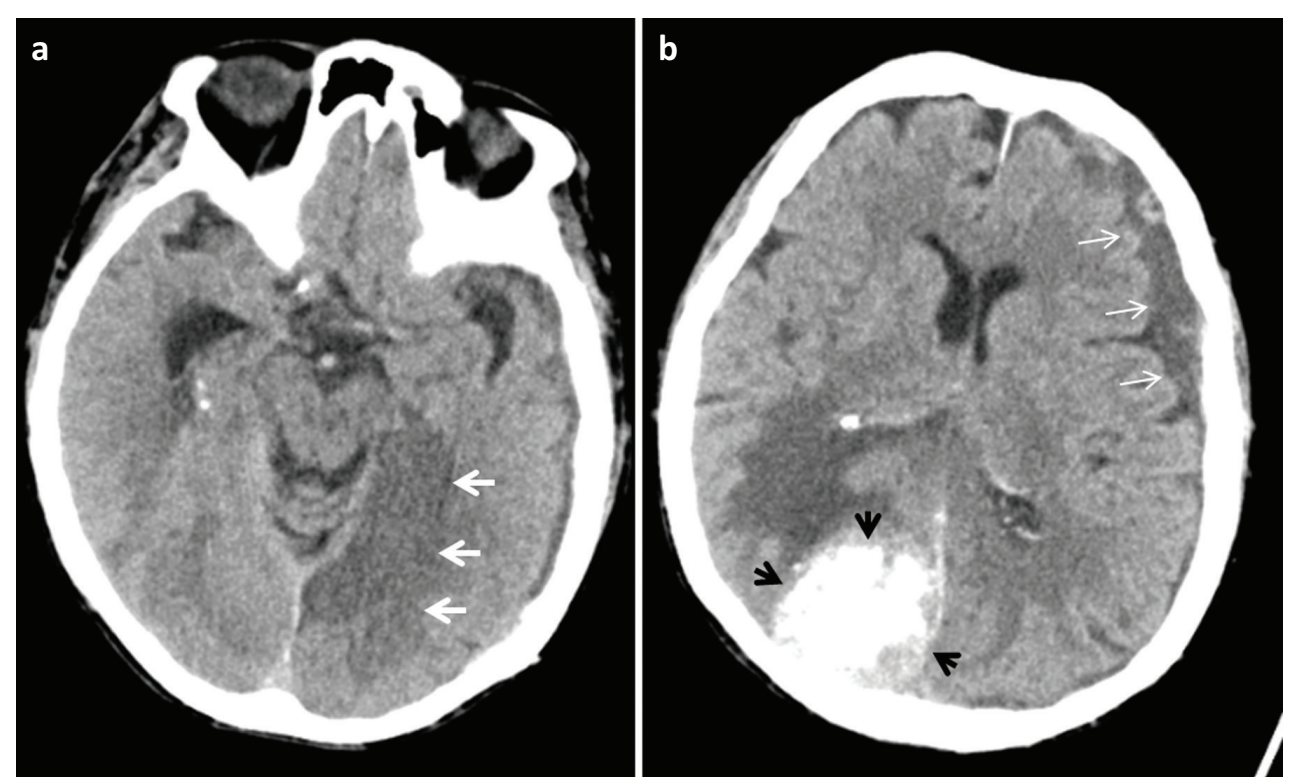

KEYWORDS: Meningioma, stroke, chronic subdural haematoma, Occam's razor, Hickam's dictum

DOI: $10.7861 /$ clinmed.2020-0039

\section{Introduction}

Finding the correct diagnosis in clinical neurology can sometimes be compared to the working method of Sherlock Holmes.' To his repertoire of skills, like competence of observation, reasoning and special knowledge, thinking tools like Occam's razor or Hickam's dictum may be added to explain clinical signs and symptoms. ${ }^{2,3}$ Occam's razor brings into focus a single explanation of a number

Authors: ${ }^{A}$ consultant in neurology, Municipal Hospital Landshut, Landshut, Germany; ${ }^{B}$ consultant in anaesthesia and emergency medicine, Municipal Hospital Landshut, Landshut, Germany; ${ }^{\mathrm{C}}$ consultant in radiology, Municipal Hospital Landshut, Landshut, Germany of complaints and clinical findings; Hickam's dictum, by contrast, delineates that there are as many diseases as the patient reports as most likely to explain the clinical condition. By presenting a case vignette of an elderly patient with initially assumed intracerebral haemorrhage, which finally turned out as multifaceted cerebral comorbidity, we wish to exemplify this idea.

\section{Case presentation}

An 87-year-old woman presented with acute visual disturbances and aphasic symptoms in the emergency department. She was on anticoagulation medication due to atrial fibrillation. The neurological examination revealed nearly complete blindness, dysphasia and pronator drift of the right limbs. As intracerebral anticoagulation-related haemorrhage was clinically assumed, cranial computed tomography (CCT) was immediately performed. CCT excluded acute haemorrhage but showed three different, hitherto unknown, distinct neuroradiological findings (Fig 1): a calcified meningioma with prominent perifocal oedema in the right occipital, an ischaemic stroke in the territory of the posterior cerebral artery and a left-sided chronic subdural haematoma. Taken together, all these findings contributed to the clinical 
decompensation of the patient's status necessitating emergency and consecutive neurohospital care.

\section{Discussion}

This case vignette highlights several points. First, multimorbidity in the elderly concerns not only different organ systems but also isolated organs, such as the brain. ${ }^{4}$ The three new detected conditions in our patient at her first presentation in the emergency room affect mainly elderly patients and, as the world population becomes older, the overall incidence of such findings will increase.

Second, the neurological examination revealed nearly complete blindness, dysphasia and pronator drift of the right limbs. Answering the pivotal question 'Where is the lesion?' these findings propose a dysfunction of both posterior brain regions and the speech-dominant left hemisphere. Due to dysphasia, however, history taking was extremely difficult, thus the onset and course of disease was difficult to gather in the first contact without a history from a third party. The imperative of skilful history taking and neurologic examination comes here to its limits and additional apparatus-supported methods like CCT, as in our patient, are necessary to clarify the clinical condition.

Third, following the idea of Occam's razor and considering the intake of oral anticoagulation, intracerebral haemorrhage would be a plausible working diagnosis in our patient. However, by CCT, a type of organ-related Hickam's dictum was found with the three aforementioned iconic findings. This case tells us that clinical reality is multifarious and ideas of Occam's razor or Hickam's dictum are both useful to find the correct diagnosis, but should not be used apodictically. Nevertheless, the art of history taking and clinical neurological examination should be foregrounded whenever feasible. ${ }^{5}$

\section{References}

1 Cherington M. Sherlock Holmes: neurologist. Neurology 1987;37:824-5.

2 Lees AJ. Noticing in neurology. Pract Neurol 2019;19:427-30.

3 Hilliard AA, Weinberger SE, Tierney LM, Midthun DE, Saint S. Clinical problem-solving: Occam's razor versus Saint's triad. N Engl J Med 2004;350:599-603.

4 Heckmann JG, Kraus ], Lang CJG. Geriatric basics for the neurologist. Neurohospitalist 2018;8:92-101.

5 Aminoff MJ. The future of the neurologic examination. JAMA Neurol 2017;74:1291-2.

Address for correspondence: Prof Josef G Heckmann, Department of Neurology, Municipal Hospital Landshut, Robert-Koch Strasse 1, 84034 Landshut, Germany. Email: josef.heckmann@klinikum-landshut.de 